\title{
Classification of models
} and description of trends in assessing the causality of relationships in socio-economic processes

\author{
Dmitry M. Nazarov \\ E-mail:slup2005@mail.ru \\ Ural State University of Economics \\ Address: 62, 8 Marta Street, Yekaterinburg 620144, Russia
}

\begin{abstract}
Scientific research of any socio-economic and managerial process can be represented as a chain of reflections on the causes and consequences of this or that phenomenon's occurrence. At the same time, the authors can try not only to answer the question "why?" but also to study and understand the nature of cause-and-effect relationships, to find out the mechanisms of their occurrence, and also to get the answer to the question posed as accurately and reasonably as possible. Each author, using the accumulated experience, offers both qualitative and quantitative methods that allow him to obtain one or another assessment of causality. However, there are not enough articles devoted to a comprehensive review of the methods and technologies of cause-and-effect relationships in socio-economic processes. This article discusses three well-known conceptual approaches to the assessment of causation in socioeconomic sciences: successionist causation, configurational causation and generative causation. The author gives his own interpretation of these approaches, builds graphic interpretations, and also offers such concepts as a linear sequence of factors, the causal field, and the causal space of factors in socioeconomic processes. Within the framework of these approaches, a classification of mathematical and instrumental models for assessing the causality of relationships in socio-economic processes is given, and trends in the development of these and new models are formulated, taking into account the global transition to a digital format. All of these trends are based on the use of digital technologies in different formats and include descriptions of such formats. The article contains specific author's examples of causality model implementation in scientific research related to economics and management.
\end{abstract}

Key words: causality; socio-economic process; sequence of reasons; configuration of reasons; generalization of reasons; causal field; causal space.

Citation: Nazarov D.M. (2020) Classification of models and description of trends in assessing the causality of relationships in socio-economic processes. Business Informatics, vol. 14, no 4, pp. 47-61. DOI: 10.17323/2587-814X.2020.4.47.61 


\section{Introduction}

$\mathrm{T}$ The issues of assessing the causality of relationships in socio-economic processes are discussed in many scientific works. At the same time, it is quite obvious that the mechanisms of the occurrence of cause-and-effect relationships are universal about the subject and object of research. Therefore, the study of cause-and-effect relationships ("causality") is given great attention in many sciences: philosophy, psychology, economics, management, physics, chemistry, etc. In socio-economic sciences, the issues of "causality" are identified with the new term "causality," which is gaining popularity. Causality (lat. causalis) - causal interdependence of events in time [1]. The variety of application spheres of the causality concept determines the diversity of approaches to its study [2-17].

Experimental and quasi-experimental methods have become the basis of research practice in the search for the causality of relationships in socio-economic processes, which have made it possible to make a real "revolution of reliability" [2] in the field of empirical socio-economic research. New methods and approaches which have gone far beyond the scope of econometric and correlation-regression analysis have made it possible to ensure high quality of the quantitative estimates obtained and to reliably identify the presence of causality, and not just correlations. This made it possible to measure accurately the strength of the impact of some observed variables on others in the framework of the socioeconomic processes under consideration.

The general meaning of the experimental approach, within which new methods of studying the causality of socio-economic processes were born, is quite simple. It consists of choosing the object of analysis, determining and formalizing the essence of the impact (influence), building a research hypothesis, as well as a comparative analysis of the impact's assessment of the selected impact (or lack of it) on groups. The most important characteristic of dividing objects into two groups (experimental and control) is the randomization of objects falling into these groups, which helps to effectively solve the problem of endogeneity. Assessment of the difference (dissimilarity) of such impacts on randomly selected groups allows us to obtain an unambiguous answer to the question of whether the selected impact is the cause of changes in the characteristics of objects. Thus, a properly constructed experimental research design is the key to a successful study of causality in socioeconomic processes.

The effectiveness of the causality study in socio-economic processes based on a wellbuilt design is confirmed by Christopher Sims and Thomas Sargent - laureates of the Nobel Prize in Economics for 2011, awarded "for empirical research of causal relationships in macroeconomics" [13, 14]. These scholars have developed methods to answer questions about the causal relationship between economic policy and various macroeconomic variables such as GDP, inflation, unemployment and investment.

When assessing causality in socio-economic processes, there are three main approaches to the study of cause-and-effect relationships in modern science.

The successionist causation approach explores and identifies vital elements of causality, such as variables or methods that describe socio-economic processes. At the same time, research is aimed at observing the relationship between such variables using survey methods, tests, and experiments. The explanation of causality is based on the differences in associative links (real or false, direct or indirect), as well as on the assessment of the strength and significance of these links [2, $3,5,12-14,18,19]$. 
The configurational causation approach implies the study of the socio-economic process based on comparison or "comparative" analysis of data. This means that research begins with the study of some cases of a certain set of socio-economic processes or phenomena that have similarities and differences. The purpose of such studies is to find causality based on the selection of two sets of factors or parameters, some of which lead to similarity and others to difference. Thus, causality in this sense is the basis for dividing the studied set of socio-economic processes into two clusters. As a result of the research, the key configurations of attributes are revealed which make it possible to explain the differences in the results for the entire set of socio-economic processes being considered [18-23].

The generative causation approach also begins by looking at measurable patterns that describe socio-economic processes. However, it is assumed that they are caused by the action of some deep mechanism that describes human actions, and, in the general case, is not formalized in the form of a set of variables or attributes. Causality, in this case, is reduced to the creation of theories of such mechanisms explaining the emergence or lack of uniformity (behavior patterns) [23-28].

The three scientific approaches presented are the basis of most scientific research aimed at elucidating causal relationships in socio-economic processes.

We should emphasize that the focus of the approaches just described is an imperative thing - "logic in use," the reason why this or that approach is applied in the first place. Each of the three approaches is based on some of the key organizational research principles that give rise to the corresponding scientific methodology. This methodology is universal and can be applied in various fields of knowledge, including social and historical sciences, pure sciences, as well as applied sciences on a macroand micro-scale.
Using the approaches discussed above, researchers of socio-economic processes are trying to solve some problems in assessing the causality of relationships between factors: the problem of the direct influence of " $\mathrm{X}$ " on "Y," the problem of delayed or retrospective causality, the problem of functionality (deterministic or probabilistic), the problem of causality of relationships and many others [2-17].

The purpose of this article is to consider the classification of causality models within the framework of the three approaches discussed above and to formulate trends in the development of causality theories concerning socioeconomic processes and phenomena, taking into account the development of tools and the transition of a society to a digital development format.

\section{Analysis of approaches to identifying causality in socio-economic processes}

In a broad sense, causality theory answers the question related to determining the truth of the statement " $\mathrm{X}$ begets $\mathrm{Y}$ ". In this case, " $X$ " is called a cause or a causal factor (or a set of factors), and " $Y$ " is called a consequence, response, or an effective factor (or a set of factors). Mathematically speaking, " $\mathrm{X}$ " is a necessary condition for " $Y$ " and " $Y$ " is a sufficient condition for " $\mathrm{X}$ " (Figure 1).

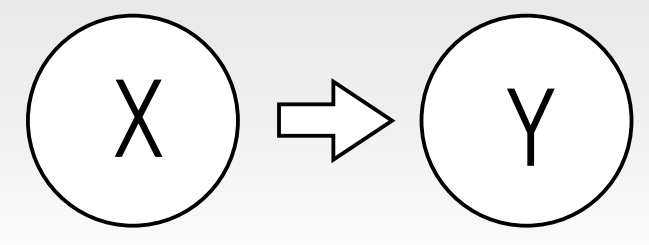

Fig. 1. Graph of factors' causality of the socio-economic process 
In the successionist causation approach, causal links are established between variables that explain the cause within a specific model describing the socio-economic process. In this case, the first step is to identify independent variables that fix the state of the process at a certain moment in time, as well as the result (effect) the dependent variable that needs to be explained. Such patterns, associations, or correlations provide the basic building blocks within the framework of the "cause-effect" dualism. However, it is well known that correlation is not causation. Therefore, within the framework of this approach, it is necessary to do a lot of work to identify the causal relationship by analyzing the data using mathematical methods. Identifying a sequence of causes avoids the fuzzy conclusion that "everything causes everything" and focuses on finding really meaningful influences. This is mainly done in two ways.

The first method is to isolate critical causal relationships by manipulating data that are randomly divided into test and control samples. In this case, all but one of the variables are fixed, and thus the strength of the influence of this variable on the result is determined. Since the samples are identical in composition, only this variable can affect the result. Thus, the direct influence of the highlighted variable on the result can be observed and measured directly.

The second way to identify causality implies a similar logic, but the author achieves control of the result differently. To examine the veracity and strength of any particular causal relationship, a test variable is introduced. The author tests this variable to see if the original influence model has changed. In other words, the strength of this variable's influence on the result and the original variable is checked.

Thus, within the framework of the first approach, the variables describing the socioeconomic process explain the obtained result. Causality is driven by incrementally adding variables, collecting data, creating measurement tools and providing capabilities for processing experimental data. At the same time, the assessment of causality is based on a deep analysis of data associated with the search for effective combinations of variables' arrays that most accurately describe the socio-economic process.

The "configurational causation" approach to identifying the causality of relationships in socio-economic processes is, in fact, based on the ideas of John Stuart Mill outlined in the book "System of Logic" [12], which were developed in [20, 21]. From a technical point of view, this is associated with the transition from a methodology based on variables to a methodology based on specific (particular) cases. From this point of view, the author considers attributes and conditions. Attributes are also identifiable through data collection. The difference is that the author considers attributes as part of the socio-economic process, and not as independent objects, but, nevertheless, they are independent variables. Thus, causality, in this sense, is determined by a special configuration of attributes in the whole system. Variable analysis in the first approach focuses on identifying the contribution of individual causes, while configuration analysis tries to trace the results based on their combination (attributes). This thesis can be schematically depicted in the following form (Figure 2).

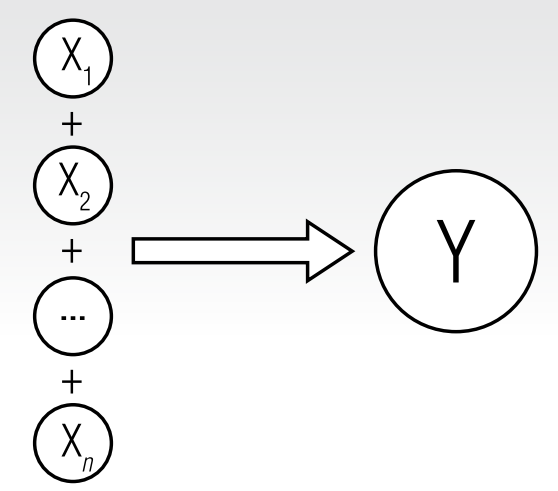

Fig. 2. Graph of factors' configurational causality of the socio-economic process 
To clarify the meaning of this graph, let us give an example of Moore's study [29], in which the early industrialization of Britain was caused by such factors as a weak aristocracy, technological progress, a strong middle class, the movement of cheap labor, the presence of colonies, etc. Any of these factors apart unlikely could cause changes in industrial production. However, taken together, these factors provide a powerful boost. Indeed, technological progress will not scale production without an influx of cheap labor, which is possible through the presence of colonies. Thus, the "+" sign in Figure 2 means the presence of a certain set of attributes. Understanding the attribute as a set of interrelated variables, we get the first fundamental feature of the second approach. Then the limitation of the first approach becomes visible, which we identified when examining a study devoted to modelling the process of Russia's accession to the WTO [30]. The main idea of configurational causality is that the change in the socio-economic process is due to the relationship of variables in the attributes. The absence of any variable in an attribute does not cause changes, consequently, the attribute itself is not the reason for this change. It is the combinatorial nature of the attribute structure that is the key characteristic of causal complexity, which the author considers in the second approach. The second important point in understanding the meaning of configurational causality is that it is comparable phenomena that are compared for the search for causality. Thus, in the example of the early industrialization of Britain, a comparative analysis of attributes' similar structure in the industrialized countries of that period (France and Germany) would give us an answer to the question of why Britain became the leader of the industrialization at that time.

Thus, in the second approach a new causal logic is established which is significantly different from the model of the first approach and has the following characteristics: $\downarrow$ attribute configurations explain the reason;

$\downarrow$ dissimilar configurations of attributes can lead to the same result;

$\downarrow$ similar configurations of attributes can lead to different results;

$\downarrow$ individual sets of attributes can lead to opposite results.

Within the framework of the second approach, a technology for identifying causal relationships was developed, which was called qualitative comparative analysis [21, 22]. This technology consists of four steps:

1. Put forward a hypothesis and select potential attributes that could lead to the investigated result;

2. Collecting data (using primary or secondary means) and placing them in a data matrix;

3. Simplification of the "truth table" to identify the most significant cause-and-effect relationships. Simplification involves deriving basic causal configurations using analytic rules, such as the following: "If two rows of a truth table differ in only one attribute, but still lead to the same result, then the attribute that distinguishes the two rows can be ignored and excluded from consideration: $\mathrm{Y}=\mathrm{X} 1 . \mathrm{X} 2 . \mathrm{X} 3 . \mathrm{X} 4$ (1100), Y = X1.X2.X3.X4 (1101)". Here "X" (uppercase letter) means code 1 (true), while " $x$ " (lowercase letter) means code 0 (false). Such a record means that the presence or absence of attribute number 4 in the truth table $\mathrm{x} 4$ (X4) has no meaning for a certain class of situations $Y=X 1 . X 2 . x 3$, if they are in the same truth table;

4. Choice of attributes' basic configurations and interpretation of results.

The generative causation approach is based on an ordered sequence of applying rules to a set of abstract symbols [1]. Issues related to the emergence and development of this approach have philosophical roots and are associated with the concepts of critical realism [25-28] and 
generative modeling [30-32]. The key difference between generative causality is the study of cause-and-effect relationships at the level of the mechanism of their occurrence and functioning in socio-economic processes (Figure 3).

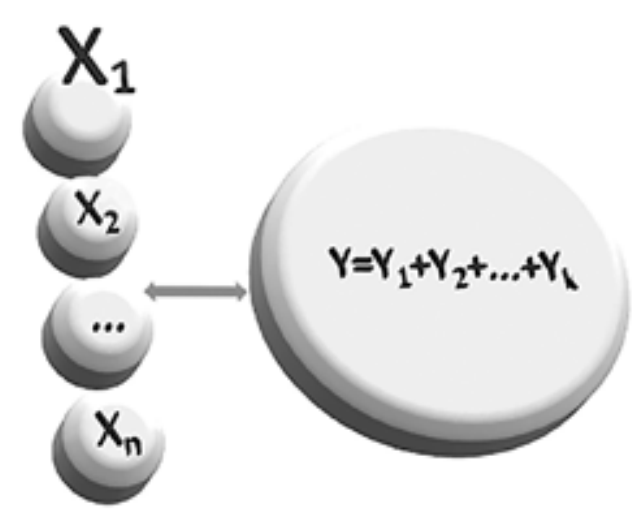

Fig. 3. Graph of factors' generative causality of the socio-economic process

The first fundamental difference of this approach lies in a different presentation of the research result. In generative explanation, the goal is to explain what causes causal relationships, that is, to identify some patterns of relationships between attributes and outcomes in the form of association rules. A set of such patterns can be considered as possible patterns of behavior in the study of socio-economic processes. In essence, the presentation of the result is also understood as a collection of attributes, and the causal arrow is replaced with an "equivalence" sign (double-headed arrow), which shows the relationship of variables' sets. Thus, in the generative approach, the results are the object of explanation, since they describe more complex sequences, comparisons, trends and relationships.

The second difference is "generative mechanisms" or "underlying mechanisms," which reflect the uniqueness of the approach and explain the patterns of results. Causal relationships, in this case, are in the "mechanism of action" of causality and are understood as "potentials" or "processes" inherent in the system under study. Thus, socio-economic research begins with templates of results and with the hypothesis about the choice and argumentation of attributes.

The third difference concerns the contexts that are needed to explain the typology of results. The contexts represent the possible options in the generative explanation of causal links. Contexts are pre-existing institutional, organizational and social conditions that define the framework for the study of causality in the socio-economic process. They allow you to develop an infrastructure for the study of causality in socio-economic processes.

Having described the features of generative causality, one can conceptually describe the search for causality in socio-economic processes. The author explains the causality of connections in socio-economic processes by the fact that the mechanism (M), acting in the context (C), will generate the result, or output $(\mathrm{O})$. This CMO technology offerings are the starting point and end product of research. The research begins with hypotheses aimed at explaining a pattern of outcomes by postulating how they can be explained within a specific context. Then the author is carrying out empirical research to understand better the setting of the action's mechanism as part of an iterative process of correlating input and output.

Thus, comparing the three listed approaches, we can state that all of them are the basis for the construction of stable informative causal links of socio-economic processes and have the property of nesting:

Sequence of causes $\subset$ Configuration of causes $\subset$ Generalization of causes 
All three approaches involve the hypotheses' construction and the interpretation of the causality's model of connections in socio-economic processes.

Within the framework of the first two approaches, researchers focus on finding and applying appropriate methods and tools to perform basic calculations that assess the strength and quality of communication, since these characteristics are fundamental for constructing the causality mechanism, which is described in the third approach. However, in the first two approaches, research has not focused on the question of why the individual partial causalities of variables and attributes make sense. This means that the variables and attributes, as well as their interrelationships, are responsible for establishing the strength of the causal relationship. But they do not take into account the infrastructure and context of the socio-economic process being considered, although these characteristics structure what is happening and affect the quality of the research. In other words, the first approach (sequence of causes), which can be conventionally called linear (one-dimensional model), can be improved by adding variables, but adding such "element-by-element" complexity leads to an increase (exponentially) in computational and descriptive complexity. The second approach (configuration of causes) takes into account the relationships between variables and allows them to be represented as attributes. Applying the provisions of this approach, we can argue that we are dealing with a causal field of factors and attributes (two-dimensional model). To improve research results in this approach, the author used additional comparisons, which also leads to an increase in the complexity of the model for assessing the relationships' causality. Within the framework of these two approaches, it is rather difficult to obtain stable empirical generalizations and explanatory persuasiveness.
The third approach (generalization of causes) has the best technology for assessing the causality of relationships in socio-economic processes since it contains elements that allow strengthening generative reasoning. However, just because "context mechanism - result" configurations provide more explanatory flexibility than variable or attribute-based models does not mean that they are in any way final or complete. Of course, formation the mechanisms of relationships causality requires a more powerful data set and complex assessment methods. At the same time, the presence of context makes it possible to strengthen the explanatory nature of causality in socio-economic processes, and the causal space of factors makes it possible to transfer the constructed mechanism from one area to another with minimal losses.

An example of generative causality can be found in the discoveries of Nobel laureates in the field of economics who build their models proceeding from some causal assumptions, obtaining new and generalizing traditional economic laws. J. Akerloff argues that rational behavior in different market segments should take into account a certain degree of information asymmetry between the seller and the buyer [34]. Thus, to study the effectiveness of market behavior (according to Pareto), it is proposed to add the attribute "information asymmetry." At the same time, the result in such a causal space turns out to be multivalued, since the attribute "asymmetry" for different categories of market agents describes the real market situation in different ways. Generative causation, in this sense, gives rise to a new theory of market behavior. The context, in this case, may be a specific market in which the above causal space of factors is considered: the used car market, insurance, and medical markets and others. 


\section{Examples of applying the approaches to identifying the causality of socio-economic processes}

\section{Example 1. Successionist causation}

In the work by M.S. Yudaeva [30], the author considers the issue of the causal link between the process of Russia's accession to the WTO and the consequences of this accession. Sequential causation here is an acyclic graph. The author proposes using the method of randomized probabilities to evaluate the strength of connections in an acyclic graph using a specific example the electric power sector of the Russian economy.

In his research, the author applied the logic of the first approach. However, the phenomenon of Russia's accession to the WTO goes far beyond the chosen variables, therefore the estimates of the impact obtained can be considered only partial (for example, no effect was found for other industries).

Despite these shortcomings, this study is as accurate as we can get close to manipulating the WTO accession process to observe the effect of one variable (the top of the acyclic graph) on another when all other variables are considered unchanged.

The basic logic of this approach remains unchanged: the author generated hypotheses about causal relationships, collected data on a set of suitable variables to study this pattern, and, in accordance with the results of the analysis, explained the alleged reason with a specific example.

\section{Example 2. Configurational causation}

The studies of the American scientist D. Meister [35] in the field of "corporate culture - profitability of an enterprise" causality reflect the principles of the configurational approach. At the first stage, the author identified several attributes of corporate culture that determine the financial success of a company: self-improvement, leadership, etc. The logic of building causal links is shown in Figure 4 . In this case, the author builds a causal field and assesses the strength of links' causality based on the results' statistical processing of 139 firms employing 5ю.589 people.

Using an econometric apparatus, D. Meister proved that two factors have the greatest influence on financial success: profit growth (0.81) and profit per employee $(0.53)$. The rest of the factors have a significantly lower level of impact ( 0.27 and 0.24 , respectively). The limitation of this study lies in the basic assumption of linearity and independence of the factors' influence.

So, the application of QCA technology in the framework of the configuration approach on specific examples of the study of the corporate culture causality and the main indicators of the enterprise (organization) showed the implementation of all the four stages indicated above. Indeed, the assessment methods developed by the authors begin with defining the structure of attributes that can contribute to the achievement of the result based on common sense, that is, the analysis of management situations in different organizations. Then the author, realizing the complexity of the phenomenon under consideration, made an attempt, each in his own way, to explain the complex interaction of attributes and build various combinations, sorting them according to their importance. Based on the configurations obtained, a method for assessing the causality of corporate culture and the main indicators of the enterprise (organization) was developed.

\section{Example 3. Generative causation}

D. Denison's model [36] of assessing the corporate culture's impact on the activities of an enterprise can be attributed to the generative approach to the generative causality study "corporate culture - the efficiency of the enterprise." 
Each component of a dual-link is a synergistic sum of several attributes. The corporate culture includes engagement, alignment, adaptability and company mission. The efficiency of the enterprise includes assets and investments, sales and product quality, employee satisfaction and the level of innovation, creativity and customer focus, sales growth and an increase in market share [36, 37].

Such decomposition allows us to present the mechanism of the relationship between corporate culture and efficiency better and contains clear signs of generative causality. This decomposition is a more accurate tool for determining the impact of corporate culture on the efficiency of an enterprise. D. Denison offered his original causal space of factors connecting the performance of an organization with corporate culture and described the mechanism of their action in various contexts.

The result of assessing the relationships' causality in D. Denison's model can be considered a set of associative relationships that generate the mechanism of corporate culture's causality and organization's performance indicators, linking attributes (components of corporate culture) and results (enterprise performance) [36, 37].

\section{Mathematical and instrumental models of causality}

The work of a fairly large number of scientists - philosophers and psychologists - was devoted to the conceptualization of causality models at the initial stages of development.

J. Mill [12] substantiated the principles of scientific knowledge and developed several conceptual models for detecting causes and effects in the study of socio-economic and other processes. He identified the understanding of the cause, using the logical interpretation of "cause is a necessary and sufficient condition of the effect," and also proposed to use the model of differences to identify causality. The essence of this model consisted of "sifting" the factors of the studied processes through the "sieve" of the criterion, which was associated with an assessment of the collinearity of the change in the premise and result.

The second most important conceptual model of causality of connections was developed by the psychologist D. Hume [38]. The basic characteristics of this model are associations, which the scientist defined as the ability to establish connections between sensations. Associations structure sensations according to the parameters of similarity and spatio-temporal extent. D. Hume defined the conditions for the emergence of the association of causality this way: cause and effect must be adjacent to each other in time and space, the cause must precede the effect and this connection must be necessary.

Thus, thanks to the conceptual models proposed in philosophy and psychology, the main factors influencing the assessment of the causal structure of processes and phenomena include the following: statistical relationships between events, the temporal order of events following each other, a change in the natural course of events as a result of different events, a-priori representations and installations.

The metric evaluating such combinations of occurrence or absence of events can be determined on the basis of the classical formalization of conceptual models - the equation of conjugation of cause and effect: $\Delta \mathrm{p}=\mathrm{p}(\mathrm{Y} \mid$ $\mathrm{X})-\mathrm{p}(\mathrm{Y} \mid \neg \mathrm{X})$. In this equation, the degree of conjugation $(\Delta p)$ is defined as the difference (according to J. Mill) of the conditional probabilities of the consequence $\mathrm{Y}$ in the presence and absence of factor $X$. Note that such formalization does not reflect the direction of the causal connection (from cause to effect), which plays a key role in assessing causality [7, $10,15,17]$. 
In mathematics, there are three well-known theories associated with modeling socio-economic processes under conditions of uncertainty: the probability theory, the theory of possibilities and the theory of fuzzy sets.

Probabilistic-statistical, fuzzy and expert methods and models are considered as fundamental economic and mathematical models of the causality of relationships between factors, implying, first of all, the study of the occurrence of events within the framework of the experiments, taking into account the design of the experimental research.

The first group of models is associated with correlation and regression analysis. Within the framework of this group of models, the structural equations and diagrams of S. Wright, the Neumann - Rubin causal models, Pearl functional models, David's dynamic models and various graph models should be noted. One way or another, in all these models, different types of correlation analysis are used as a measure of determination, and the correlation coefficient is calculated. More detailed information on such models is contained, for example, in works [5, 9, 39-43].

The second group of models has received the general name "confirmatory analysis" [44, 45]. The essence of confirmatory factor analysis is to assess the strength of relationships for some latent variable that affects the results and attributes. This approach, based on the econometric apparatus of dependence studies, allows one to compare different structures of factors. The methodology of confirmatory analysis is based on deductive logic, therefore this method is popular in assessing the causality of relationships.

The third group of models is based on expert methods for assessing various parameters of socio-economic processes based on the analysis and comparison of multi-criteria alternatives. The essence of matrix expert methods consists of the construction of matrices that reflect the relative importance of the investigated alternatives in different forms for a given pool of features. As for graph methods, they, first of all, involve the construction of some kind of hierarchical (or network) structure that reflects mutual influences and implements routes to achieve various goals. Note that the proposed division is conditional since both graph and matrix methods can use separate elements of each other simultaneously. Speaking about the features of each method, we should pay attention to the algorithms of expert assessments that answer the question: how is the opinion of an expert or a group of experts processed? Among these algorithms, at least two groups can be distinguished - algorithms for quantitative and qualitative assessments. The most common methods of expert relationships' causality assessment are methods that allow one to evaluate various coefficients of causal relationships between factors of socio-economic processes: DEMATEL [45-47], MICMAC [48-51], as well as a method for detecting and assessing the influence of implicit factors [39-41].

The DEMATEL method (Decision Making Trial and Evaluation Laboratory) [45-47] is one of many multicriteria decision-making methods that implies the effective identification of causal relationships of a complex system based on the aggregation of expert assessments. This method aggregates the collective expert opinion to exclude random relationships between indicators and criteria and, based on causal links, identify the most important indicators that determine the integral characteristic. The method allows you to determine the direct, reverse and indirect relationships, as well as the direction of the interdependence between the criteria and indicators.

The MICMAC method [48] stands for "Matrix d'Impacts Croises Multiplication 
Appliqué un Classement" which literally means a composition of the cross matrix and classification. The analysis using this method is a procedure for constructing a classification matrix of the factors' cross-influence and is intended to assess the degree of dependence of the variables' influence (the strength of causal links) based on ranking. Each of the factors studied belongs to one of four clusters: autonomous, dependent, interrelated and independent. These factors (drivers) are grouped based on a specific potential and strength of influence. Autonomous factors (quadrant 1) are factors that have weak potential and strength of influence. As a rule, they are practically insignificant in determining causality. Dependent factors (quadrant 2) are factors that have a low potential but strong influence. Interrelated factors (quadrant 3) are factors that have high potential and power to influence. These factors are causally related, which means that an action on one of them will lead to a change in the other. Independent factors (quadrant 4) are factors that have strong potential but little impact. All factors are plotted on a four-cluster graph, where the potential of the variable is on the $\mathrm{Y}$-axis and the force of influence is on the $\mathrm{X}$-axis.

In the works [39-41, 52], a pool of economic and mathematical models is proposed that allows, on the study's basis of organizational management factors' variety, to single out implicit ones. After this, based on the apparatus of fuzzy logic, using the Gauguin's implication, to assess the influence's degree of these factors on other factors' management. As an example, it is proposed to assess the impact of corporate culture on the main indicators of the organization's performance. At the same time, using fuzzy binary relations, it is possible to obtain a causal field of factors that determine the causal relationship "corporate culture - the main indicators of the organization's activities."

\section{The main trends in causality model development of socio-economic processes}

Modern digital technologies make it possible to receive and process large amounts of data in real-time. This makes it possible to widely use the arsenal of mathematical theories and methods associated with probabilistic, statistical and expert assessment of various determinants of socio-economic processes.

Accordingly, there are at least three main trends that will facilitate a "revolution of reliability" in causal studies of socio-economic processes and improve the quality of their management.

The first trend is associated with the development of existing methods based on the aggregation of estimates obtained using big data processing technologies. This trend implies the development within the framework of all three approaches to assess causality; however, it most clearly codifies the first two approaches - the sequence of causes and the configuration of causes. Indeed, the methods of data mining, firstly, are "not afraid" of a multiple increase in variables in causal models of the study of socio-economic processes. This means that using the available tools (SAP Analytics Cloud, SAP HANA, Power BI, QlikView, Phyton, R, etc.), the researcher, given the data, can perform multiple types of checks on various factors for causality and increase the reliability of the result. Secondly, given the availability of data, it is possible to build algorithms for formalizing quantitative estimates (for example, ranking factors) that will partially or completely replace expert opinion.

The second trend is associated with the use of intelligent data processing algorithms that can be configured to "measure" the causality of fields and factor spaces. This trend will significantly formalize the approach of generalization of causes and make it accessible to most researchers. Note that the generalization of 
causes implies the construction of associations and classifications (according to D. Hume and J. Mill). Within the framework of existing algorithms, The author would like to pay attention to two main methods - the algorithm for constructing Bayesian networks and the Apriori algorithm, which, based on specially prepared data sets, allow us to construct association rules characterizing the behavioral characteristics of people - participants of socio-economic processes.

Over the past twenty years, Bayesian networks have become one of the basic tools for formalizing uncertainties in artificial intelligence. Bayesian networks not only provide a natural and compact way to encode factors of exponential size in causal space but also give efficient probabilistic inference in realtime [53-56]. It is important that Bayesian networks are directed acyclic graphs, where nodes are random variables, and edges are conditional relationships between random variables, distributed either discretely or continuously. Since most of the structural and functional models of socio-economic processes are presented in the form of various hierarchies (acyclic graphs), then Bayesian networks are ideal for identifying the causality of relationships in them.

The Apriori algorithm [56] and its modifications allow one to formalize the so-called recommender systems, within the framework of which associative rules are constructed based on data in the form of "if-then" implications. Different data slices and sets of variables make it possible to establish the causality of relationships in different contexts of socio-economic research. The essence of the algorithm makes it possible to drastically reduce the dimension of data, but its settings and speed of action in realtime - to carry out a large number of experiments.

The third trend is related to machine learning algorithms and methods. Due to the flex- ibility of settings and instrumental support, these algorithms allow, within the framework of any of the above approaches, to develop new methods and technologies for assessing causal links in socio-economic processes that are not known today.

\section{Conclusion}

Within the framework of the theoretical and methodological study of generally accepted approaches, an attempt was made to study the causality of socio-economic processes in world science, to build their models based on a deep analysis of the content on this issue. It should be noted that there are clearly not enough publications in Russian science that use the three main approaches to the study of cause-and-effect relationships, which include successionist causation, configurational causation and generative causation. The digital format of the development of socio-economic processes allows one to go from conceptualizing causality directly to the applied use of the accumulated experience and knowledge in this area, using modern tools for analyzing big data. This approach will make it possible to more accurately identify the cause-and-effect relationships of social processes and get a better effect from research in this direction. The versatility of the methods and models considered guarantees the successful application of software for the development of research in the field of building applied digital models of causality in socio-economic processes.

\section{Acknowledgments}

This article was supported by the Russian Foundation for Basic Research, within the framework of the Russian Foundation for Basic Research competition "Expansion-2019" No 19-110-50281. 


\section{References}

1. Ilyichev L.F., Fedoseev P.N., Kovalev S.M., Panov V.G., eds. (1989) Philosophical encyclopedic dictionary. 2-nd ed. Moscow: Soviet Encyclopedia (in Russian).

2. Angrist J.D., Pischke J.-S. (2010) The credibility revolution in empirical economics: How better research design is taking the con out of econometrics. Journal of Economic Perspectives, vol. 24, no 2, pp. 3-30. DOI: $10.1257 /$ jep.24.2.3.

3. Almond D., Mazumder B. (2011) Health capital and the prenatal environment: The effect of Ramadan observance during pregnancy. American Economic Journal: Applied Economics, vol. 3, no 4, pp. 56-85. DOI: 10.1257/app.3.4.56.

4. Asher H.B. (1983) Voting behavior research in the 1980s: An examination of some old and new problem areas. Political science: The state of the discipline (ed. A.W. Finifter). Washington DC: American Political Science Association, pp. 339-388.

5. Dawid A.P. (2012) The decision-theoretic approach to causal inference. Causality: Statistical perspectives and applications (eds. C.R. Berzuini, A.P. Dawid, L. Bernardinelli), chapter 4. Wiley and Sons, pp. 25-42. DOI: 10.1002/9781119945710.ch4.

6. Glymour C.N. (2001) The mind's arrows: Bayes nets and graphical causal models in psychology. Cambridge, MA: MIT Press.

7. Mackie J.L. (1974) The cement of the universe: A study of causation. Oxford, England: Clarendon Press.

8. Murphy G.L. (2004) The big book of concepts. Cambridge, MA: MIT Press.

9. Pearl J. (2009) Causality models, reasoning, and inference. Cambridge: Cambridge University Press.

10. Rescorla R.A., Wagner A.R. (1972) A theory of Pavlovian conditioning: Variations in the effectiveness of reinforcement and non-reinforcement. Classical conditioning II: Current research and theory (eds. A.H. Black, W.F. Prokasy). New York: Appleton-Century-Crofts, pp. 64-99.

11. Steyer K., Simon O., Kraus R.H.S., Haase P., Nowak C. (2013) Hair trapping with valerian-treated lure sticks as a tool for genetic wildcat monitoring in low-density habitats. European Journal of Wildlife Research, no 59, pp. 39-46. DOI: 10.1007/s10344-012-0644-0.

12. Mill J.S. (2002) A system of logic. Honolulu: University Press of the Pacific.

13. Sargent T.J. (1979) A note on maximum likelihood estimation of the rational expectations model of the term structure. Journal of Monetary Economics, no 5, pp. 133-143.

14. Sims C. (1972) Money, income and causality. American Economic Review, vol. 62, no 4, pp. 540-552.

15. Suppes P. (1973) A probabilistic theory of causality. British Journal for the Philosophy of Science, vol. 24, no 4, pp. 409-410.

16. Salmon W. (1984) Scientific explanation and the causal structure of the world. Princeton, N.J.: Princeton University Press.

17. Woodward J. (2003) Making things happen: A theory of causal explanation. Oxford: Oxford University Press.

18. Campbell D., Stanley J. (1963) Experimental and quasi-experimental designs for research. Chicago: Rand McNally.

19. Moore B. (1966) Social origins of dictatorship and democracy. Boston: Beacon Press.

20. Skocpol T., ed. (1984) Vision and method in historical sociology. Cambridge: Cambridge University Press.

21. Ragin C. (1987) The comparative method. Berkeley: University of California Press.

22. Ragin C. (1994) Constructing social research. Thousand Oaks: Pine Forge Press.

23. Abbott A. (1998) The causal devolution. Sociological Methods and Research, vol. 27, no 2, pp. 148-181.

24. Archer M. (1995) Realist social theory. Cambridge: Cambridge University Press.

25. Bhaskar R. (1978) A realist theory of science. Hassocks: Harvester Press. 
26. Bhaskar R., Archer M., Collier A., Lawson T., Norrie A. (1988) Critical realism: Essential readings. London: Routledge.

27. Blalock H. (1961) Causal inferences in non-experimental research. Chapel Hill: University of North Carolina Press.

28. Pawson R. (1989) A measure for measures: A manifesto for empirical sociology. London: Routledge.

29. Moore B. (1966) Social origins of dictatorship and democracy. Boston: Beacon Press.

30. Gorban M.I., Guriev S.M., Yudaeva K.V. (2002) Russia in the WTO: myths and reality. Voprosy Ekonomiki, no 2, pp. 61-82 (in Russian).

31. Boudon R. (2006) Tocqueville for today. Oxford: Bardwell Press.

32. Elster J. (1989) Nuts and bolts for the social sciences. Cambridge: Cambridge University Press.

33. Fararo T. (1989) The meaning of general theoretical sociology: Tradition and formalization. Cambridge: Cambridge University Press.

34. Akerlof G.A. (1970) The market for "lemons": Quality uncertainty and the market mechanism. Quarterly Journal of Economics, no 84, pp. 488-500.

35. Maister D.H. (2001) Practice what you preach: What managers must do to create a high achievement culture. New York: Free Press.

36. Denison D., Hooijberg R., Lane N., Lief C. (2012) Leading culture change in global organizations: Aligning culture and strategy. San Francisco, CA: Jossey-Bass.

37. Denison Consulting (2009) Culture getting started guide. Available at: http://staff.studentlife.umich.edu/ files/dsa/Getting+Started+Guide.pdf (accessed 24 May 2020).

38. Hume D. (1965) A treatise on human nature. Essays in 2 vols. Vol. 2. Moscow: Myisl (in Russian).

39. Nazarov D.M. (2017) Fuzzy model for assessment of causality of factors in collaborative economy. Proceedings of the 2017 IEEE 19th Conference on Business Informatics (CBI 2017), Thessaloniki, Greece, 24-27 July 2017, vol. 2, pp. 28-31. DOI: 10.1109/CBI.2017.4.

40. Nazarov D.M. (2019) The fuzzy logic methodology for evaluating the causality of factors in organization management. Open access peer-reviewed chapter. Available at: https://www.intechopen.com/books/ fuzzy-logic/the-fuzzy-logic-methodology-for-evaluating-the-causality-of-factors-in-organizationmanagement (accessed 01 July 2020). DOI: 10.5772/intechopen.84814.

41. Nazarov D.M. (2016) Methodology for fuzzy-multiple assessment of implicit factors in the organization's activities. Ekaterinburg: USUE (in Russian).

42. Wright S. (1921) Correlation and causation. Journal of Agricultural Research, no 20, pp. 557-585.

43. Rubin D.B. (1978) Bayesian inference for causal effects: The role of randomization. Annals of Statistics, vol. 6 , no 1 , pp. 34-58.

44. Hair J.F., Hult G.T.M., Ringle C.M., Sarstedt M. (2017) A primer on partial least squares structural equation modeling (PLS-SEM). Thousand Oaks, CA: SAGE Publications.

45. Jassbi J., Mohamadnejad F., Nasrollahzadeh H. (2011) A fuzzy DEMATEL framework for modeling cause and effect relationships of strategy map. Expert Systems with Applications, vol. 38, no 5, pp. 5967-5973. DOI: 10.1016/j.eswa.2010.11.026.

46. Chen F.H., Chi D.-J. (2015) Application of a new DEMATEL to explore key factors of China's corporate social responsibility: Evidence from accounting experts. Quality \& Quantity, vol. 49, no 1, pp. 135-154. DOI: $10.1007 / \mathrm{s} 11135-013-9978-2$.

47. Wu H.-H., Chang S.-Y. (2015) A case study of using DEMATEL method to identify critical factors in green supply chain management. Applied Mathematics and Computation, vol. 256, pp. 394-403. DOI: $10.1016 / \mathrm{j}$.amc.2015.01.041.

48. Khanam S., Siddiqui J., Talib F. (2016) Modeling the TQM enablers and IT resources in the ICT industry: An ISM-MICMAC approach. International Journal of Information Systems and Management, vol. 1, no 3, pp. 195-218. DOI: 10.1504/IJISAM.2015.072290. 
49. Mudgal R., Shankar R., Talib P., Raj T. (2009) Greening the supply chain practices: An Indian perspective of enablers' relationship. International Journal of Advanced Operations Management, vol. 1, no 2-3, pp. 151-176. DOI: 10.1504/IJAOM.2009.030671.

50. Poduval P.S., Pramod V., Raj J. (2015) Interpretive structural modeling (ISM) and its application in analyzing factors inhibiting implementation of total productive maintenance (TPM).

International Journal of Quality \& Reliability Management, vol. 32, no 3, pp. 308-331. DOI: 10.1108/IJQRM-06-2013-0090.

51. Raj T.; Shankar R.; Suhaib M. (2008) An ISM approach for modelling the enablers of flexible manufacturing system: The case for India. International Journal of Production Research, vol. 46, no 24, pp. 6883-6912. DOI: 10.1080/00207540701429926.

52. Nazarov D.M. (2016) Theoretical and methodological foundations of fuzzy-multiple assessment of implicit factors of organization management. Doctoral thesis. Saint-Petersburg (in Russian).

53. Russell S., Norvig P. (1995) Artificial intelligence: A modern approach. Prentice Hall.

54. Suwignjo P., Bititci U.S., Carrie A.S. (2000) Quantitative models for performance measurement system. International Journal of Production Economics, vol. 64, no 1-3, pp. 231-241.

DOI: $10.1016 /$ S0925-5273(99)00061-4.

55. Tian J. (2005) Identifying direct causal effects in linear models. Proceedings of the 20th National Conference on Artificial Intelligence (AAAI-05), Pittsburgh, Pennsylvania, 9-13 July 2005, vol. 1, pp. 346-352.

56. Agrawal R., Srikant R. (1994) Fast algorithms for mining association rules in large databases. Proceedings of the 20th International Conference on Very Large Data Bases, Santiago, Chile, 12-15 September 1994, pp. 487-499.

\section{About the author}

\section{Dmitry M. Nazarov}

Dr. Sci. (Econ.);

Head of the Department of Business Informatics, Institute of Management and Information Technologies, Ural State University of Economics, 62, 8 Marta Street, Yekaterinburg 620144, Russia;

E-mail: slup2005@mail.ru 